\title{
Role of ultrasound scanning in community contraceptive and reproductive health clinics
}

\author{
Andrea Brockmeyer, Anne Webb
}

\begin{abstract}
Background The role of ultrasound scanning (USS) in the community setting of contraception and reproductive health $(\mathrm{CRH})$ has not yet been clearly established. Abacus Clinics use ultrasound scans prior to referral for abortion where gestation is uncertain and for location of an intrauterine device/intrauterine system (IUD/IUS).
\end{abstract}

Objective To audit the indications for USS performed in Abacus Clinics and to determine a minimum acceptable number of annual scans for each clinician.

Methods A retrospective review of case notes was performed for a period of 3 months.

Results The majority of women (36; $64 \%$ ) had a scan to determine gestation prior to referral for abortion. In 12 $(21 \%)$ women the scan was performed for location of an IUD/IUS. Two (4\%) women had a scan for both indications. Only six (11\%) women did not comply with the indications listed above but all were related to gestation or
IUD/IUS use. In 16 women the scan significantly influenced decision-making regarding referral to the appropriate abortion service provider. The number of scans performed by individual doctors varied between two and 12.

Conclusions The use of USS prevented unnecessary referrals to hospital and delays in clients' care. The number of scans each clinician performed was small and the indications for future USS were only slightly changed. The new special skills module in ultrasound developed by the Faculty of Sexual and Reproductive Healthcare (FSRH) (formerly the Faculty of Family Planning and Reproductive Health Care) aims to set new standards and should improve training.

Keywords community settings, special skills module, training, ultrasound scanning

J Fam Plann Reprod Health Care 2008; 34(2): 115-117

(Accepted 24 September 2007)

\section{Introduction}

In the UK, ultrasound scanning (USS) is frequently performed in hospital gynaecology. To date, the use of USS in the community setting of contraception and reproductive health $(\mathrm{CRH})$ has not been established. Reasons for this are the uncertainty of indications, limited availability of scanning equipment and lack of experienced sonographers. Abacus Clinics in Liverpool, UK has nearly 60000 client visits per year, mostly for community sexual health services, with a small gynaecological component. There has been an ultrasound scanner at its main clinic for several years.

In Liverpool there is pressure on hospital scanning facilities with long waits for appointments to check for intrauterine devices (IUD) or systems (IUS) with lost threads. In addition, induced abortions are carried out by two different service providers, depending on gestation. If referred to the inappropriate provider, a woman could incur significant delays before treatment. Accurate determination of gestation as early as possible obviates some of these problems.

The clinic doctors who scan were trained in a variety of settings. Some had considerable experience from backgrounds in general obstetrics and gynaecology. One doctor had done a short course in transvaginal scanning only. Two more underwent a local course with a half-day theory session and 20 one-to-one practical sessions, mostly in antenatal booking and gynaecology clinics.

The clinical protocols of Abacus Clinics identified just two indications for USS within the service:

\footnotetext{
Abacus Clinics for Contraception and Reproductive Health, Liverpool, UK

Andrea Brockmeyer, MRCOG, MFSRH, Subspecialty Registrar

Anne Webb, Mrcog, FFSRH, Consultant

Correspondence to: Dr Andrea Brockmeyer, Central Abacus, Contraception and Reproductive Health Services, Citrus House, 40-46 Dale Street, Liverpool L2 5SF, UK.

E-mail: andrea.brockmeyer@liverpoolpct.nhs.uk
}

\section{Key message points}

- Ultrasound scanning (USS) in a contraceptive and reproductive health setting can reduce hospital referrals and improve continuity of care.

- Training and maintenance of skills are essential.

- National recommendations on training for USS in community settings have recently been published.

- to determine gestation prior to referral for abortion where uncertainty of gestation may lead to referral to an inappropriate service provider or

- to locate an IUD or IUS.

An audit was proposed to determine whether the USS performed at Abacus Clinics adhered to these indications, to assess whether they needed changing, and identify if individual clinicians were able to maintain skills by undertaking a 'reasonable' number of ultrasound scans within the community service.

\section{Methods}

A retrospective audit was performed over a period of 3 months. Computerised records were analysed to determine the number of scans performed during this period. Clients' records were reviewed to find out the indications for USS and the outcomes. These were then compared with the standards set in the clinical protocols.

\section{Results}

The computerised records identified 63 ultrasound scans. Three of the women for whom a scan was recorded were initially referred to hospital and did not have a scan within Abacus Clinics. The computerised record does not differentiate between the locations where a scan is carried out. One scan was recorded four times in error. In one case neither an indication for a scan nor a report was found within the clinical records. Overall, 56 case records were assessed in detail.

The scans were carried out by six doctors, who were 
Table 1 Initial indications for ultrasound scanning

\begin{tabular}{lc}
\hline Indication & Scans $(\boldsymbol{n})$ \\
\hline Gestation & 37 \\
Prior to abortion & 36 \\
Viability in planned pregnancy & 1 \\
IUD/IUS & 17 \\
Location of IUD/IUS only & 12 \\
Other indications & 5 \\
Gestation prior to abortion and IUD/IUS location & 2 \\
\hline
\end{tabular}

IUD, intrauterine device; IUS, intrauterine system.

present for two to eight clinics per week. The number of scans each doctor performed over the 3-month period varied between two and 12. Thirty-two women had a transvaginal scan, 17 had a transabdominal scan and seven had both.

Table 1 shows that 36 women had a scan to determine gestation prior to abortion referral. One woman had pain early in a planned pregnancy and was referred on to the hospital emergency department after the scan. In 12 women the scan was performed only for location of an IUD/IUS. Two women were pregnant and needed IUD location. Five women with an IUD/IUS were outside the agreed indications: in three women a scan was performed at the time of a difficult IUD fitting; in one a scan was performed for pelvic pain a month after IUD insertion; and in another a scan was done at the client's request because she could not feel the threads and was not reassured enough by being told that they were seen. Whilst trying to identify the presence or absence of an IUD two pelvic masses were seen and the women appropriately referred on to hospital.

Of the 38 clients for whom determination of gestation prior to referral for abortion was the main indication, 32 were directly referred for abortion without the need for a second scan. For 16 of them, the result of the scan significantly influenced decision-making, allowing more appropriate referral to the relevant service provider. One woman requested an abortion and had a scan but decided to think about the abortion again and did not re-attend the clinic.

Six women who requested abortion had gestations clinically over 20 weeks. The scan at Abacus confirmed the late gestation but the clinician had enough uncertainty to request a confirmatory scan at the hospital. One woman decided after her initial scan to continue with the pregnancy without the need for a second scan. Three more continued with their pregnancy after their hospital scan (one was over the 24-week legal limit) whereas two women underwent late abortion outside the region, as there is no provision for late abortions locally.

A total of 30 of the 56 women would have required a hospital scan if they had not had one in the community and five others would probably also have been referred. In the remainder the scan supported the planned management and helped to reduce anxiety associated with uncertain gestation.

All the scans were accurate in determining whether the IUD/IUS was in its correct position. In one case a second hospital scan was requested, which confirmed the original findings.

\section{Audit outcome}

As a result of this audit, and following discussion, some changes were made to the clinical protocols:

- All clinicians performing USS at Abacus Clinics should have had formal training in carrying out the procedures and been assessed to be competent by the clinician with lead responsibility for USS in the service.

- Each clinician performing USS should perform a minimum of 12 scans per year.

- For women with a gestation greater than 16 weeks, a confirmatory second scan should be considered at the local hospital unless it is expected that the woman will be seen by the local abortion service provider for assessment within the next 7 days.

- The indications for performing USS at Abacus Clinics remain broadly the same, although for women requesting abortion USS can be performed if the gestation is in any doubt or if the outcome of the scan may change the management in any way.

- If any pathology is found on USS that is not related to IUD/IUS location or gestation prior to referral for abortion then the woman should be managed by appropriate onward referral.

- All clinicians performing USS should keep a logbook of all the scans performed and audit their performance using any available information in the notes such as letters following abortion or results of other scans.

A re-audit following the introduction of these new guidelines is planned in 12 months.

\section{Discussion}

USS is used widely in hospital settings but rarely in the community. Abacus Clinics introduced the use of USS for limited indications within sexual health care.

USS in general can be very helpful in aiding the diagnostic process, but unhelpful or even dangerous if wrongly interpreted or performed by inexperienced clinicians. Most senior gynaecologists were taught by a 'hands on' approach and had little structured training. Conversely, they gained a huge amount of clinical experience because of their volume of work. The Royal College of Obstetricians and Gynaecologists has introduced USS into its basic logbook for trainees. Most trainees will attend a basic ultrasound course but access to clinical experience is variable. For other clinicians it is more difficult to find enough practical experience and clinicians able to carry out the necessary assessments. There is also a shortage of sonographers to deliver the service, let alone any training.

The results of our audit show that the use of USS prior to referral for abortion and for location of IUD/IUS reduces the number of referrals to hospital and allows for better continuity of care.

Could the indications be widened further? A recent article by Iskaros $^{1}$ argues that USS can often reduce the need for pelvic examination. We concur with him that training is a vital issue and, at present, although there are a number of theoretical and introductory courses for USS, not all of them focus on the skills most useful within a CRH setting. After all, the main indication in this situation is not trying to identify pathology, but gestation prior to referral for abortion and the presence or absence of an IUD/IUS. There will still need to be a link with the specialist service to follow up unexpected findings.

The results of our audit also showed that doctors performed a variable number of ultrasound scans. In view of the limited indications for USS in our service, it was agreed to allow a small recommended minimum number of scans annually. This may need to be reviewed in future in the light of the Faculty of Sexual and Reproductive Healthcare (FSRH) (formerly the Faculty of Family Planning and Reproductive Health Care) recommendations. The recently developed FSRH special skills module in ultrasound ${ }^{2}$ recommends that a clinician should perform a minimum of 30 scans a year to maintain 
standards. However, the module covers many more indications than those currently agreed in Abacus Clinics. There is no doubt that the skills needed to identify a lost subcutaneous implant are different to those required for transvaginal scanning. Most of the scans performed in $\mathrm{CRH}$, however, are going to be unplanned and the need identified during the consultation. Therefore, as they will mainly involve early pregnancy or lost IUD threads, transvaginal scanning skills are essential.

There will also always be situations in which a further scan performed by a more experienced sonographer will be necessary. These may include those women considering abortion at later gestations. Although the numbers are small, it is obviously essential to ensure that gestations are within the legal limit and to assist in the decision on the unit to which the women should be referred, as there are very few centres within the UK that will perform late abortions and consequently any women in this position may have to travel long distances. Accurate determination of gestation will avoid wasted time and travel as well as delays that may limit women's choices.
If a CRH service were to provide outpatient gynaecology then the possible uses of USS would be greater, but training and knowledge would also need to be wider and it is unlikely that anyone who had not undergone full gynaecological USS training would provide such a service.

The special skills module for ultrasound developed by the FSRH sets new standards. We hope that its recommendations will lead to specific training, including the practical element, focused on the needs of clients attending $\mathrm{CRH}$ services in the community.

\section{Statements on funding and competing interests Funding None identified. \\ Competing interests None identified.}

\section{References}

1 Iskaros J. Gynaecological ultrasound in the community. J Fam Plann Reprod Health Care 2006; 32: 71-73.

2 Syllabus and Logbook for the Certificate in Ultrasound of the Faculty of Sexual and Reproductive Healthcare of the Royal College of Obstetricians and Gynaecologists. September 2006. http://www.fsrh.org/pdfs/UltrasoundLogbook.pdf [Accessed 15 September 2007].
Obesity and Reproductive Health. P Baker, A Balen, L Poston, N Sattar (eds). London, UK: RCOG Press, 2007. ISBN: 1-90475 239-4. Price: $£ 48.00$. Pages: 286 (paperback)

The prevalence of obesity in adult women in the $\mathrm{UK}$ is rapidly increasing and is currently approaching $25 \%$. Besides having significant unwanted consequences on the health of the population, obesity has a negative impact on fertility and may lead to potential problems in pregnancy. This text, produced by an RCOG Study Group of experts in the field, provides a comprehensive review of the effects of obesity on various issues concerned with reproductive health.

The book provides a wealth of largely evidence-based information on the management of obese women with menstrual disturbances and/or infertility, including an excellent chapter on polycystic ovary syndrome. The importance of lifestyle improvement is emphasised. The maternal, fetal and obstetric complications of maternal obesity are addressed. The use of hormonal contraceptives in overweight women with additional risks is carefully discussed.

This well-written text would be of value to health care professionals involved in the practice of reproductive health and also to other clinicians with an interest in obesity.

Reviewed by Ruzva Bhathena, MD, FRCOG Consultant Obstetrician and Gynaecologist, B D Petit Parsee General and Masina Hospitals, Bombay, India

Vulvovaginal Infections. WJ Ledger, SS Witkin. London, UK: Manson Publishing Ltd, 2007. ISBN: 1-84076-069-9. Price: £40.00. Pages: 128 (hardback)

American authors William J Ledger (not to be confused with his British namesake) and Steven Watkin are eminent professors in the field of gynaecological infection. Their depth of experience and enthusiasm for the subject is evident in this interesting and very readable textbook. With 128 pages and an attractive hardback cover, the book was slim and discreet enough to read on my daily commute to work. However, the inside pages are so vividly illustrated with clinical pictures that I soon abandoned this idea.

There are 15 chapters, all well referenced.
The book begins with an overview of vaginal microbiology and immunology. The scientific detail is clear and concise, with helpful diagrams and frequent reference to points of clinical relevance. Microbiological nomenclature is forever changing and immunology has moved on in leaps and bounds since I was an undergraduate, so I found this a useful update. There follows a chapter on history taking, examination and investigation, including interpretation of vaginal $\mathrm{pH}$ testing and near-patient microscopy.

The main body of the book deals with the various infective diseases of the vulva and vagina, both sexually and non-sexually transmitted. Each chapter is divided into microbiology, immunology, diagnosis and treatment. Allergic vulvovaginitis, desquamative inflammatory vaginitis, vulval pain and dermatological diseases are also covered. I looked eagerly for pearls of wisdom on the management of recurrent candidal infection. There are interesting explanations as to why some individuals may be predisposed to recurrent candidal infection or resistant to treatment, but I was disappointed to find little new in terms of management. Although sexually transmitted infections are covered in reasonable depth the book is not intended for the genitourinary medicine specialist.

I found this book illuminating and very well illustrated. By relating the clinical picture to the underlying pathophysiology the authors succeed in simplifying what is often a difficult clinical problem. The book will be of interest to clinicians in the field of women's health or general practice, particularly those with a special interest in infection or vulval disease.

Reviewed by Louise Melvin, MRCOG, MFSRH Subspecialty Specialist Registrar in Sexual and Reproductive Health, Edinburgh, UK

Birth Control, Sex and Marriage in Britain 1918-1960. K Fisher. Oxford, UK: Oxford University Press, 2006. ISBN: 0-19926-736-7. Price: $£ 50.00$. Pages: 300 (hardback)

This fascinating book grew out of in-depth interviews with 193 women and men born between 1899 and 1933 and from a variety of social backgrounds. They were asked about their attitude to and practice of birth control within their marriages. It is known that during the early years of the last century family size reduced dramatically and while there was a post-war increase in the numbers of children born, families never reverted to the late Victorian size. We also know that Marie Stopes and other birth control pioneers were active in promoting contraception and setting up clinics. It is easy to assume that women would have been delighted to take control of their fertility and be released from endless pregnancies and child rearing. These interviews tell a different story.

Most women interviewees claimed a high degree of ignorance about the new appliance methods of birth control: they identified themselves as naive and sexually passive and sought to preserve this state. To actively seek contraception threatened their view of themselves. When women did use birth control they preferred to use methods that did not require any forward planning, or negotiation, or preparation for sex. They might therefore seek out abortion, and many interviewees had some knowledge about both folk methods, such as slippery elm, and illegal surgical methods.

It was assumed in many marriages that the man should and would take care of contraception, and they did. Despite the availability of condoms and caps, withdrawal was a hugely popular method. Many of the men interviewed prided themselves on their self-control and successfully used this method for family planning over many years. When withdrawal was the main method there was still a feeling that babies came along 'naturally' and many couples rejected appliance methods as being cold, calculating and against the natural order.

Families were rarely 'planned' with any discussion between man and wife: it was man's role as head of the household to make these decisions, whether he saw himself as a traditional authoritarian husband or a more modern companionate husband. Most women interviewed expected and accepted this authority from their husbands.

This book would interest anyone interested in social history and particularly the way gender roles influenced the uptake of new methods of contraception. It challenges assumptions about what women want and about the roles and attitudes of men and provides a glimpse of the vanishing world of Britain in the early 20th century.

Reviewed by Clare Payne, FFSRH

Associate Specialist in Reproductive Health

Care, North Devon, UK 